\title{
The Clinical Significance of Cancer Stem Cell Markers ALDH1A1 and CD133 in Lung Adenocarcinoma
}

\author{
TAKEAKI MIYATA ${ }^{1,2}$, TSUNEHIRO OYAMA ${ }^{1,3}$, TAKASHI YOSHIMATSU ${ }^{2}$, HANAE HIGA $^{2}$, \\ DAIGO KAWANO $^{2}$, ATSUSHI SEKIMURA ${ }^{4}$, NAOKI YAMASHITA ${ }^{5}$, TETSUYA SO $^{5}$ and AKINOBU GOTOH ${ }^{1}$ \\ ${ }^{1}$ Laboratory of Cell and Gene Therapy, Hyogo College of Medicine, Nishinomiya, Japan; \\ ${ }^{2}$ Department of Thoracic Surgery, Fukuoka-Wajiro Hospital, Fukuoka, Japan; \\ ${ }^{3}$ Imamitsu Home Care Clinic, Kitakyusyu, Japan; \\ ${ }^{4}$ Department of Thoracic Surgery, Shin-Takeo Hospital, Takeo, Japan; \\ ${ }^{5}$ Department of Thoracic Surgery, Shin-Komonji Hospital, Kitakyusyu, Japan
}

\begin{abstract}
Background/Aim: Aldehyde dehydrogenase-1Al (ALDH1A1) and CD133 have been identified as markers of cancer stem cells (CSCs). We investigated the expression of these markers and their clinical significance in lung adenocarcinoma. Materials and Methods: An immunohistochemical analysis of ALDHIA1 and CD133 expression of 92 lung adenocarcinomas was performed. The association between the expression of both markers and cancer-related death and recurrence was determined. Results: Cancerrelated death and tumor recurrence were observed in 15 and 17 cases, respectively. The expression of CD133, but not ALDHAlA, was significantly associated with poorer overall survival $(p<0.0001)$ and shorter disease-free interval $(D F I)$ $(p<0.0001)$. Multivariate analysis revealed that double negativity was independently associated with increased survival (hazard ratio $(H R)=16.1, p=0.0004$ ) and a longer DFI (HR=9.5, $p=0.0007)$. Conclusion: We propose that as a functional marker, ALDHIAl positivity may influence the viability of CSCs. The findings suggest that it is important to evaluate the expression of both markers.
\end{abstract}

Despite continuous efforts to improve therapeutic response, lung cancer is the most common cause of cancer-related deaths in Japan, with adenocarcinoma by far the most common histology, accounting for nearly $70 \%$ of lung cancer

This article is freely accessible online.

Correspondence to: Takeaki Miyata, Institute for Advanced Medical Sciences, Hyogo College of Medicine, Laboratory of Cell and Gene Therapy, 1-1, Mukogawa-cho, Nishinomiya-City, Hyogo, Japan. Tel: +81 798456808, Fax: +81 798456806, e-mail: t.miyata.gts@gmail.com

Key Words: Lung adenocarcinoma, ALDH1A1, CD133, cancer stem cells, CSCs. cases (1). Cancer stem cells (CSCs) have the ability for selfrenewal and multipotently differentiate (2). CSCs may be highly resistant to chemotherapy and radiation, and be responsible for tumor initiation, progression and metastasis $(3,4)$. Accumulating evidence supports the existence of a CSC phenotype in human lung cancer $(3,5-7)$. The metabolic marker aldehyde dehydrogenase isoform 1A1 (ALDH1A1) and the cell-surface marker CD133 are reported to be markers of lung CSCs (3). We, therefore, investigated ALDH1A1 and CD133 expression in a retrospective cohort of 92 patients with lung adenocarcinoma. The objectives were to determine the association between ALDH1A1 and CD133 expression in lung adenocarcinoma, the relationship between their expression and the clinichopathological parameters, and the prognostic value of ALDH1A1 and CD133.

\section{Materials and Methods}

Patient population and clinicopathological data. We examined a series of 154 Japanese patients with lung adenocarcinoma who underwent surgical treatment at Fukuoka-Wajiro Hospital, Fukuoka, Japan from 2006-2012. Ninety-two out of 154 (59.7\%) patients were selected based on the following inclusion criteria: (i) no chemotherapy or radiotherapy before surgery, and (ii) the availability of an adequate paraffin block and clinicopathological data for the analysis. Follow-up information was obtained from the patients' records. Routinely collected clinicopathological data included age, gender, smoking history and pathological stage. There were 49 males (53\%) and 43 females (47\%), with a median age of 71 years (range $=40-92$ years) at the time of surgery; $43(47 \%)$ patients were $<70$ years and $49(53 \%)$ were $\geq 70$ years of age. Half of the patients $(n=46)$ had a smoking history. The pathological stages were: stage $\mathrm{I}, \mathrm{n}=62(67.4 \%)$; stage II, $\mathrm{n}=14$ (15.2\%); stage III, $\mathrm{n}=12(13.0 \%)$; and stage IV, $\mathrm{n}=4(4.4 \%)$ (TNM staging of lung cancer) (8). The median follow-up period was 1,702 days (range=4-3679 days). Recurrence was diagnosed by computed tomography alone or combined with positron-emission tomography. 
Specimen characteristics. All tissue specimens were formalin-fixed and paraffin-embedded according to routine diagnostic procedures. Hematoxylin and eosin (H\&E)-stained slides and medical records were reviewed to confirm the diagnosis according to the new International Association for the Study of Lung Cancer, American Thoracic Society and European Respiratory Society International Multidisciplinary Lung Adenocarcinoma Classification (9).

Evaluation of immunohistochemical staining. Immunohisto-chemical (IHC) staining of the 92 lung adenocarcinoma sections for ALDH1A1 and CD133 was performed using a standard immunoperoxidase technique by two investigators in a coded manner without previous knowledge of the clinical or pathological parameters. In difficult cases, the score was confirmed by two observers and a consensus was achieved. The analysis was performed using direct light microscopy, only staining specific to cancer cells was considered positive (staining of stromal tissue, macrophages and cellular debris was considered nonspecific and was excluded).

ALDH1A1 staining and scoring. ALDH1A1 staining was performed on 4- $\mu$ m-thick paraffin sections using a mouse monoclonal antibody(anti-ALDH1; Abcam Inc., Tokyo, Japan) at 1:200 dilution (Figure 1A). Normal human liver sections were used as positive controls. Negative controls were made by replacing the primary antibodies with Tris-buffered saline. ALDH1A1 immunoreactivity was scored in the cytoplasmic compartment, but not on pericellular membranes. The cytoplasmic staining intensity was scored based on the immunoreactivity as: 0 (negative), 1 (weak), 2 (intermediate) or 3 (strong). The percentage of positively stained cells in the tumor section was graded as $0-100 \%$ (at 10\% intervals). The ALDH1A1 score was assigned to each case by multiplying the staining intensity score by the percentage of stained cells as follows: ALDH1A1 score $=(\%$ of cells of intensity $1 \times 1)+(\%$ of cells of intensity $2 \times 2)$ $+(\%$ of cells of intensity $3 \times 3)$. ALDH 1 A 1 positivity was defined by an ALDH1A1 score of $>100$.

CD133 staining and scoring. CD133 staining was performed on 4$\mu \mathrm{m}$-thick paraffin sections using a mouse monoclonal antibody (antiCD133; Millipore Inc., Temecula, CA, USA) at 1:200 dilution (Figure 1B). The CD133 expression was defined as the proportion of cells in the tumor section with strong membranous staining within the neoplastic epithelial component. The percentage of positively stained cells was graded as $0-100 \%$ (at $5 \%$ intervals). When $\geq 10 \%$ of the tumor was stained it was considered CD133-positive. CD133 antigen was used as a positive control. Negative controls were made by replacing the primary antibodies with Tris-buffered saline.

Statistical analysis. All data were analyzed using the statistical software StatView (SAS Institute Inc. Cary, NC, USA). The correlation between ALDH1A1 score, CD133 expression and the clinicopathological parameters was evaluated using unpaired $t$-tests for statistical significance. Relationships between the ALDH1A1/CD133 phenotype and the clinicopathological parameters were evaluated using unpaired $t$-tests for statistical significance.

Overall survival (OS) was defined as the duration (in days) between surgery and cancer-related death (in-hospital death within 30 days after surgery and non-cancer-related deaths were excluded). The disease-free interval (DFI) was defined as the duration (in days) between surgery and the first recurrence of cancer. Patients who survived with or without recurrence were censored on the date of the last follow-up examination. The OS and DFI curves were calculated by using the Kaplan-Meier method and were examined by using the $\log$-rank test. Univariate and multivariate analyses were also performed using Cox's proportional hazards model to determine the factors that significantly influenced survival and DFI. $p$-Values of 0.05 or less were considered to indicate statistical significance.

\section{Results}

Patient characteristics. Fifteen (16.3\%) cancer-related deaths occurred (pathological stage I-IV: 5/62, 4/14, 3/12, 3/4, respectively) and tumor recurrence was observed in 17 (18.4\%) cases (pathological stage I-IV: 6/62, 4/14, 4/12,3/4) among the 92 patients with lung adenocarcinoma. Male sex, being a current or former smoker, and pathological stage IIIV disease were associated with shorter OS than the female sex, never smoking and pathological stage I disease ( $p=0.0233, p=0.044$ and $p=0.0021$, respectively; data not shown). Patients with pathological stage II-IV disease had a significantly higher recurrence rate than those with pathological stage I disease $[p=0.0018$; pathological stage I: 6/62 (9.6\%), II-IV:11/30 (36.6\%)].

Relationship between the ALDHIAl score, clinical parameters and expression of CD133. The mean ALDH1A1 score of the 92 patients was 52.1; there were therefore $20(21.7 \%)$ ALDH1A1-positive cases. No significant relationships were observed between ALDH1A1 positivity, clinicopathological parameters and expression of CD133 (data not shown). ALDH1A1 positivity was not related to clinicopathological parameters or CD133 expression (data not shown).

There was no significant difference in the curves for OS ( $p=0.1529)$ or DFI $(p=0.3156)$ by ALDH1A1 status (Figure $2 \mathrm{~A}$ and $\mathrm{B})$. A multivariate analysis that included ALDH1A1 status, CD133 expression and pathological stage revealed that ALDH1A1 status had no significant impact on OS $(p=0.8077)$ or DFI $(p=0.7652)$ (Table I).

Relationship between the expression of CD133 and clinical parameters. The median CD133 staining percentage was $3.52 \%$. There were 18 (19.5\%) CD133-positive cases. The rate of CD133 positivity in patients with pathological stage II-IV disease was significantly higher rate than those with pathological stage I $[p=0.0040$; pathological stage I: $7 / 62$ (11.2\%), II-IV: $11 / 30(36.6 \%)]$

Figure $2 \mathrm{C}$ and D show the OS and DFI curves for CD133negative and -positive cases. CD133 positivity had a significant impact on both OS $(p<0.0001)$ and the DFI $(p<0.0001)$. A multivariate analysis that included the ALDH1A1 score, the expression of CD133 and the pathological stage revealed that $\mathrm{CD} 133$ positivity was independently associated with decreased survival $(p<0.0001$, $\mathrm{HR}=20.0)$ and a decreased DFI [hazard ratio $(\mathrm{HR})=13.6$, $p<0.0001]$ in patients with lung adenocarcinoma (Table I). 


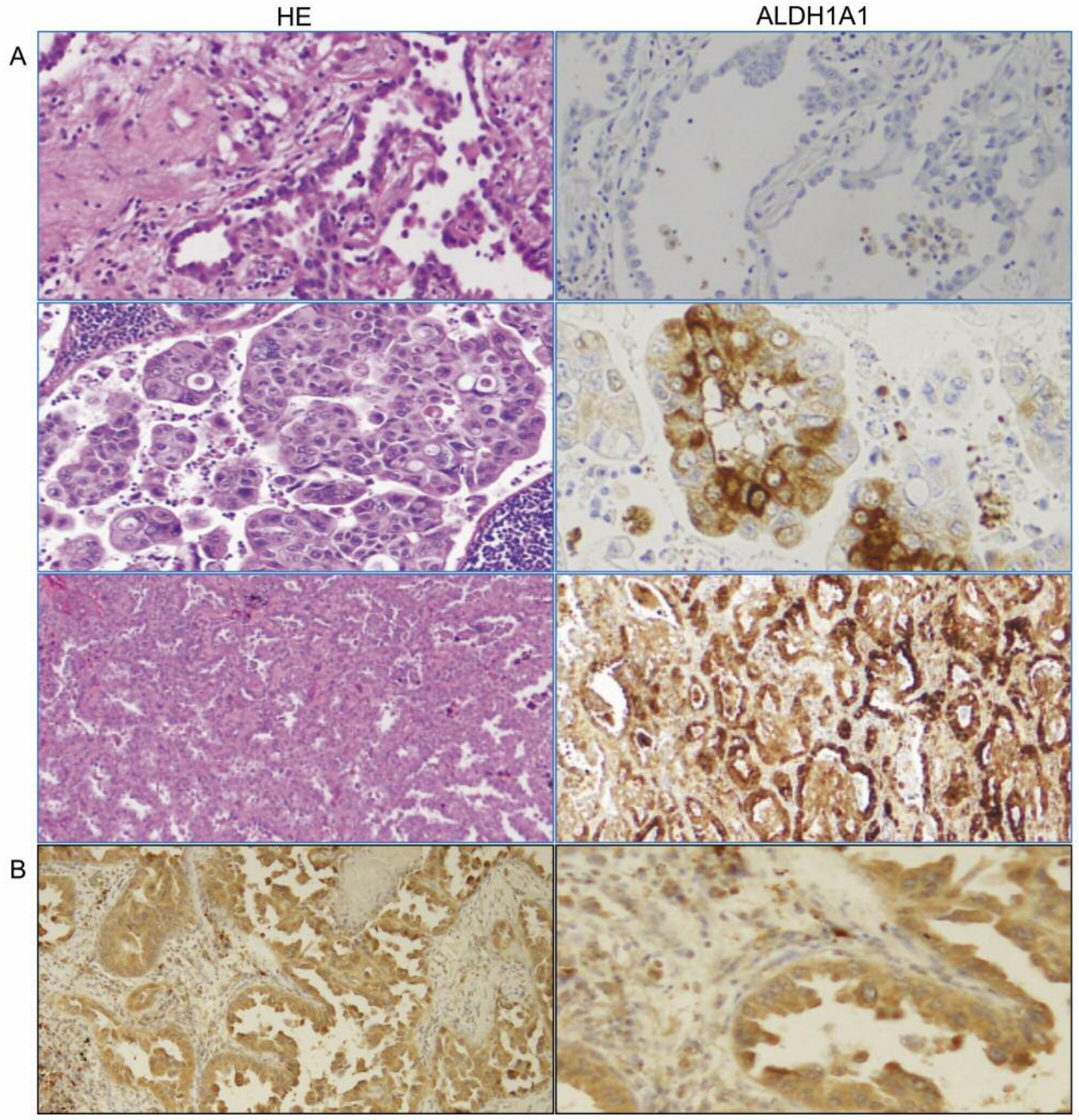

Figure 1. The validation of immunohistochemical staining of aldehyde dehydrogenase-1A1 (ALDH1A1) (A) and CD133 (B). A: The intensity of cytoplasmic staining of ALDH1A1 was scored based on immunoreactivity as 0 (negative), 1 (weak), 2 (intermediate) or 3 (strong). Intensity score and the percentage of positivity stained cells were then multiplied to give the final score. Upper row, ALDH1A1 score $=0$ (intensity $0 \times 0 \%$ positive cells). Middle row, ALDH1A1 score $=90$ (intensity $2 \times 30 \%$ positive cells + intensity $3 \times 10 \%$ positive cells). Lower row, ALDH1A1 score $=270$ (intensity $3 \times 90 \%$ positive cells). B: Example of CD133 expression: $10 \%$ staining. CD133 staining was defined as the proportion of cells with strong membranous expression (i.e. 0-100\%). Magnification: 100× (left column) and 200× (right column).

Combined analysis of the ALDH1A1/CD133 status. The patients were divided into four categories/three groups based on their ALDH1A1 and CD133 expression levels:
ALDH1 A1 $1^{-} / \mathrm{CD}_{133^{-}} \quad(\mathrm{n}=59 ;$ double-negative group $)$ $\mathrm{ALDH} 1 \mathrm{~A} 1^{+} / \mathrm{CD}_{133^{-}} \quad(\mathrm{n}=15)$ and $\mathrm{ALDH} 1 \mathrm{~A} 1^{-} / \mathrm{CD} 133^{+}$ $\left(\mathrm{n}=13\right.$; any-positive group); and $\mathrm{ALDH} 1 \mathrm{~A} 1^{+} / \mathrm{CD} 133^{+}(\mathrm{n}=5$; 

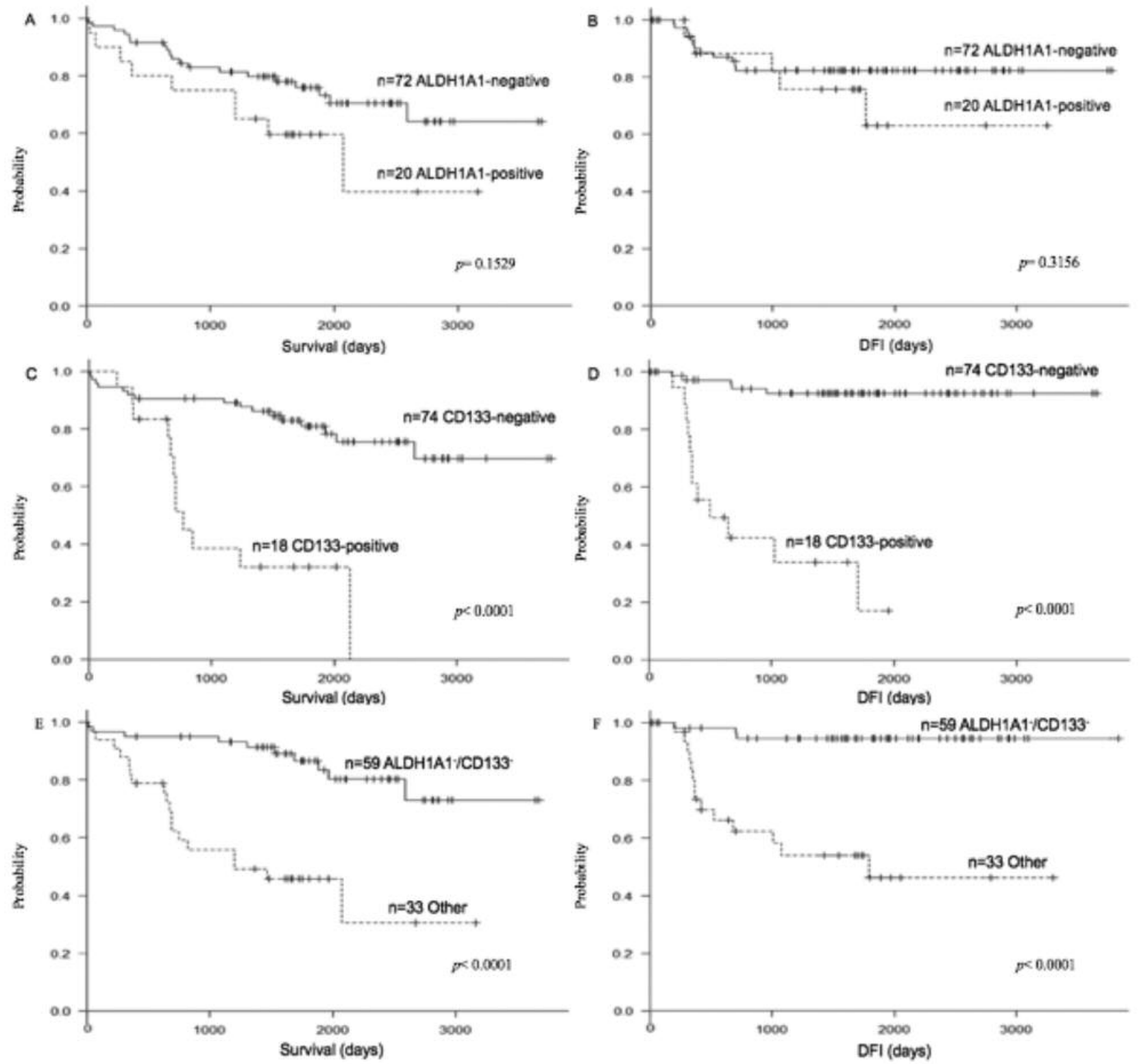

Figure 2. The Kaplan-Meier analysis of overall survival $(A, C, E)$ and disease-free interval $(D F I)(B, D, F)$ in patients with lung adenocarcinoma according to aldehyde dehydrogenase-1A1 (ALDH1A1) (A, B), CD133 (C,D) and combined ALDHA1/CD133 (E, F) expression. ALDH1A1 positivity was defined as an ALDH1A1 score of $>100$ (median was 52.1). CD133 positivity was defined as $\geq 10 \%$ cells with strong membranous expression.

double-positive group). There was no relationship between the ALDH1A1 score and the expression of CD133 ( $p=0.4886$; data not shown). The combined analysis of the clinicopathological parameters of the four categories was performed. In patients with ALDH1 $\mathrm{A}^{-} / \mathrm{CD} 133^{-}$disease, $74.2 \%$ had pathological stage I, which was significantly higher than the percentage in the ALDH1 $1^{-} / \mathrm{CD} 133^{-}$ category $(43.3 \%)$ who were mainly classified as having pathological stage II-IV ( $p=0.0038$; date not shown).
Most patients were classified into the double-negative group, which had a significantly better prognosis according to the OS and DFI curves (Figure 2E and F) (both $p<0.0001$ ). In contrast, the double-positive group had a significantly worse prognosis than the other groups $(p=0.0096$ and $p=0.0345$, respectively) (data not shown). The multivariate survival and DFI analyses revealed that double-negativity was also independently associated with improved OS $(p=0.0004$, $\mathrm{HR}=16.1)$ and DFI $(p=0.0007, \mathrm{HR}=9.5)$ (Table I). 
Table I. Univariate and multivariate analyses using a proportional hazards model to investigate the parameters associated with overall survival $(O S)$ and the disease-free interval (DFI) in patients with lung adenocarcinoma (n=92). Univariate and multivariate analyses were carried out to determine survival according to aldehyde dehydrogenase-1A1 (ALDH1A1) and CD133 expression, and pathological stage. Bold typeface indicates statistical significance $(p<0.05)$.

\begin{tabular}{|c|c|c|c|c|c|c|c|c|c|}
\hline \multirow[b]{2}{*}{ Parameter } & & \multirow[b]{2}{*}{ Subgroup } & \multirow[b]{2}{*}{$\mathrm{n}$} & \multicolumn{3}{|c|}{ Univariate analysis } & \multicolumn{3}{|c|}{ Multivariate analysis } \\
\hline & & & & HR & $95 \% \mathrm{CI}$ & $p$-Value & HR & $95 \% \mathrm{CI}$ & $p$-Value \\
\hline \multicolumn{10}{|l|}{ OS } \\
\hline \multirow[t]{6}{*}{ Model 1} & ALDH1A1 & Negative & 72 & 1 & & & 1 & & \\
\hline & & Positive & 20 & 2.1 & $0.157-1.365$ & 1.629 & 0.8 & $0.362-3.691$ & 0.8077 \\
\hline & CD133 & Negative & 74 & 1 & & & 1 & & \\
\hline & & Positive & 18 & 24.3 & $0.013-0.134$ & $<0.0001$ & 20.0 & $0.013-0.191$ & $<0.0001$ \\
\hline & Pathological stage & I & 62 & 1 & & & 1 & & \\
\hline & & II-IV & 30 & 4.5 & $0.076-0.653$ & 0.0062 & 1.7 & $0.173-1.908$ & 0.3658 \\
\hline \multirow[t]{4}{*}{ Model 2} & ALDH1A1/CD133 & Double-negative & 59 & 1 & & & 1 & & \\
\hline & & Other & 33 & 20.8 & $0.011-0.216$ & $<0.0001$ & 16.1 & $0.013-0.290$ & 0.0004 \\
\hline & Pathological stage & I & 62 & 1 & & & 1 & & \\
\hline & & II-IV & 30 & 4.5 & $0.076-0.653$ & 0.0062 & 2.0 & $0.161-1.498$ & 0.2114 \\
\hline \multicolumn{10}{|l|}{ DFI } \\
\hline \multirow[t]{6}{*}{ Model 1} & ALDH1A1 & Negative & 72 & 1 & & & 1 & & \\
\hline & & Positive & 20 & 1.6 & $0.208-1.675$ & 0.3212 & 0.8 & $0.388-3.622$ & 0.7652 \\
\hline & CD133 & Negative & 74 & 1 & & & 1 & & \\
\hline & & Positive & 18 & 16.6 & $0.020-0.175$ & $<0.0001$ & 13.6 & $0.022-0.237$ & $<0.0001$ \\
\hline & Pathological stage & I & 62 & 1 & & & 1 & & \\
\hline & & II-IV & 30 & 4.3 & $0.084-0.617$ & 0.0036 & 2.1 & $0.160-1.391$ & 0.1731 \\
\hline \multirow[t]{4}{*}{ Model 2} & ALDH1A1/CD133 & Double-negative & 59 & 1 & & & 1 & & \\
\hline & & Other & 33 & 12.3 & $0.023-0.282$ & $<0.0001$ & 9.5 & $0.029-0.387$ & 0.0007 \\
\hline & Pathological stage & I & 62 & 1 & & & 1 & & \\
\hline & & II-IV & 30 & 4.3 & $0.084-0.617$ & 0.0036 & 2.2 & $0.160-1.275$ & 0.1333 \\
\hline
\end{tabular}

HR: Hazard ratio; CI: confidence interval.

\section{Discussion}

The human ALDH enzyme family comprises of 19 members (10). ALDH, which has several isoforms (mainly ALDH1A1, ALDH1A2, and ALDH1A3), is a marker of normal tissue SCs and CSCs, in which it is involved in self-renewal, differentiation and self-protection (10). ALDH1 is expressed in putative lung epithelial SC niches and is overexpressed in tumors in comparison to normal lung (11). The ALDH1 isoforms (including ALDH1A1) play a role in retinoic acid formation through the oxidation of all-trans-retinal and 9cis-retinal, which are involved in retinoid signaling, which is related to the stemness of CSCs and normal tissue SCs (10-13). ALDH1A1 activity also has a known role in drug resistance $(10,14)$ and radioresistance $(15)$.

The biological behavior of isolated ALDH-positive lung cancer cells was observed to be highly tumorigenic and clonogenic in comparison to their ALDH-negative counterparts and the cells were capable of self-renewal (5, $16,17)$. A recent meta-analysis showed that the increased expression of ALDH1A1 is associated with poor OS and disease-free survival in lung cancer $(5,16-19)$.
Although we assumed that ALDH1A1 was associated with a poor prognosis in lung adenocarcinoma, ALDH1A1 positivity had no significant impact on OS and DFI in our study. We hypothesize that two factors affected this result. Firstly, our ALDH1A1 score was determined by multiplying the intensity score by the percentage of stained cells (similarly to other researchers) (19). The cut-off level of our ALDH1A1 scoring system was 100 ; this was chosen because it was approximately double the median ALDH1A1 score (52.1). There is therefore room for considering use of a different cutoff level. Secondly, our study population was small, which might have prevented some results from reaching significance. Further studies in larger cohorts are needed.

The CD133 antigen is a pentaspan transmembrane glycoprotein $(120 \mathrm{kDa})$ of 865 amino acids and is the product of a single-copy gene on chromosome 4 (4p15.33) in humans $(3,20)$. Its biochemical function has gradually been clarified. When cells or tissues are damaged by chemical, physical or mutational causes, CD133-positive progenitor or SCs are activated to self-renew, proliferate and differentiate to repair the damage. CD133-positive CSCs are responsible for tumor metastasis, chemoresistance, radioresistance and recurrence 


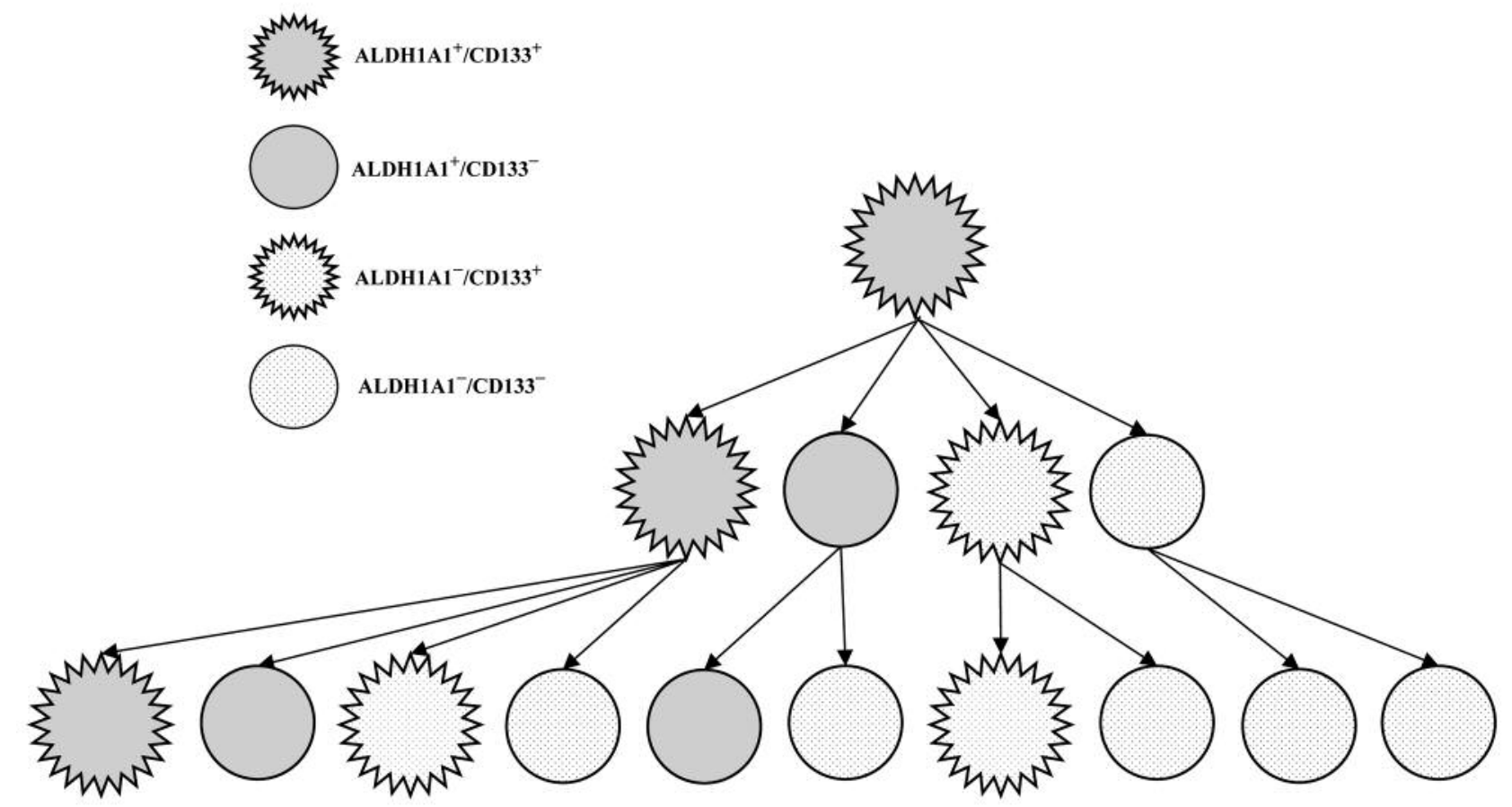

Figure 3. The model of aldehyde dehydrogenase-1A1 (ALDH1A1)/CD133 phenotypes and the prognosis of each patient group. A schematic diagram of the self-renewal and multi-potent differentiation of cells with ALDH1A1/CD133 phenotypes. The double-positive group had numerous active cancer stem cells (CSCs, spiky symbol); this group had the worst prognosis. The ALDH1A1+/CD133- group had few active CSCs. The $A L D H 1 A 1^{-} / C D 133^{+}$had numerous dormant CSCs. Thus, the any marker-positive group $\left(A L D H 1 A 1^{+} / C D 133^{-}\right.$and $\left.A L D H 1 A 1^{-} / C D 133^{+}\right)$had an intermediate prognosis. The double-negative group had few dormant CSCs. This group had the best prognosis.

(21). The expression of CD133 is dynamic and responds to changes in the cell microenvironment. CD133 is involved in diverse cellular processes, including the uptake of glucose and transferrin, autophagy, membrane-membrane interaction, and matrix metalloproteinase functions (21). The expression of $\mathrm{CD} 133$ on the cell surface was shown to be a specific marker for CSCs in a number of malignancies, including lung cancer $(6,22)$. Although several landmark studies have revealed that isolated CD133-positive lung cancer cells exhibited in vitro CSC-like features and in vivo tumorigenicity, subsequent studies showed similar tumorigenic potential of both CD133-positive and CD133negative lung cancer cells both in vitro and in vivo $(6,23)$. In previous studies, the expression of CD133 was correlated with a poor prognosis in lung cancer (24). Similarly to other reports $(24,25)$, our analysis indicated that CD133-positive tumors were associated with significantly worse outcomes in comparison to CD133-negative tumors. We therefore consider CD133 to be an appropriate CSC marker.

Previous studies investigated the relationship between ALDH1A1 and CD133 $(18,26,27)$. The results of these studies differed. Although the ALDH1A1 score and the expression of CD133 were unrelated in our analysis, the double-negative group had the best prognosis based on the
OS and DFI curves (Figure 2E and F). Furthermore, the multivariate analyses confirmed that double-negativity was independently associated with improved OS and DFI (Table I). Although there is room for debate regarding the direct relationship between ALDH1A1 and CD133, with regard to the measurement of the viability of CSCs, the findings suggest that it is important to evaluate both CD133 as a surface marker of CSCs and ALDH1A1 as a functional marker. Figure 3 illustrates the clinical significance of ALDH1A1 and CD133 as CSC markers in lung adenocarcinoma. ALDH1A1 and CD133 can both be considered as novel diagnostic and therapeutic markers for targeted lung adenocarcinoma therapy.

\section{Acknowledgements}

The Authors thank the Department of Pathology at Fukuoka-Wajiro Hospital for providing specimens and technical support.

\section{References}

1 Okumura M: Trends and current status of general thoracic surgery in Japan revealed by review of nationwide databases. J Thorac Dis 8(Suppl 8): S589-S595, 2016.

2 Lee JT and Herlyn M: Old disease, new culprit: tumor stem cells in cancer. J Cell Physiol 213(3): 603-609, 2007. 
3 O'Flaherty JD, Barr M, Fennell D, Richard D, Reynolds J, O'Leary J and O'Byrne K: The cancer stem-cell hypothesis: its emerging role in lung cancer biology and its relevance for future therapy. J Thorac Oncol 7(12): 1880-1890, 2012.

4 Diehn M, Cho RW, Lobo NA, Kalisky T, Dorie MJ, Kulp AN, Qian D, Lam JS, Ailles LE, Wong M, Joshua B, Kaplan MJ, Wapnir I, Dirbas FM, Somlo G, Garberoglio C, Paz B, Shen J, Lau SK, Quake SR, Brown JM, Weissman IL and Clarke MF: Association of reactive oxygen species levels and radioresistance in cancer stem cells. Nature 458(7239): 780-783, 2009.

5 Jiang F, Qiu Q, Khanna A, Todd NW, Deepak J, Xing L, Wang H, Liu Z, Su Y, Stass SA and Katz RL: Aldehyde dehydrogenase 1 is a tumor stem cell-associated marker in lung cancer. Mol Cancer Res 7(3): 330-338, 2009.

6 Eramo A, Lotti F, Sette G, Pilozzi E, Biffoni M, Di Virgilio A, Conticello C, Ruco L, Peschle C and De Maria R: Identification and expansion of the tumorigenic lung cancer stem cell population. Cell Death Differ 15(3): 504-514, 2008.

7 Ho MM, Ng AV, Lam S and Hung JY: Side population in human lung cancer cell lines and tumors is enriched with stem-like cancer cells. Cancer Res 15;67(10): 4827-4833, 2007.

8 Goldstraw P, Crowley J, Chansky K, Giroux DJ, Groome PA, Rami-Porta R, Postmus PE, Rusch V and Sobin L: The IASLC Lung Cancer Staging Project: proposals for the revision of the TNM stage groupings in the forthcoming (seventh) edition of the TNM Classification of malignant tumours.; International Association for the Study of Lung Cancer International Staging Committee; Participating Institutions. J Thorac Oncol 2(8): 706714, 2007.

9 Travis WD, Brambilla E, Noguchi M, Nicholson AG, Geisinger K, Yatabe Y, Powell CA, Beer D, Riely G, Garg K, Austin JH, Rusch VW, Hirsch FR, Jett J, Yang PC, Gould M and American Thoracic Society. International Association for the Study of Lung Cancer/American Thoracic Society/European Respiratory Society: international multidisciplinary classification of lung adenocarcinoma: executive summary. Proc Am Thorac Soc 8(5): 381-385, 2011.

10 Tomita H, Tanaka K, Tanaka $\mathrm{T}$ and Hara A: Aldehyde dehydrogenase $1 \mathrm{~A} 1$ in stem cells and cancer. Oncotarget 8;7(10): 11018-11032, 2016.

$11 \mathrm{Li} \mathrm{X}$, an WL, Geng J, Wu CL and Bai X: Aldehyde dehydrogenase $1 \mathrm{~A} 1$ possesses stem-like properties and predicts lung cancer patient outcome. J Thorac Oncol 7: 1235-1245, 2012

12 Patel M, Lu L, Zander DS, Sreerama L, Coco D and Moreb JS: ALDH1A1 and ALDH3A1 expression in lung cancers: correlation with histologic type and potential precursors. Lung Cancer 59(3): 340-349, 2008.

13 Liang D and Shi Y: Aldehyde dehydrogenase-1 is a specific marker for stem cells in human lung adenocarcinoma. Med Oncol 29(2): 633-639, 2012

14 Suresh R, Ali S, Ahmad A, Philip PA and Sarkar FH: The role of cancer stem cells in recurrent and drug-resistant lung cancer. Adv Exp Med Biol 890: 57-74, 2016.

15 Zhang J, Zhang C, Hu L, He Y, Shi Z, Tang S and Chen Y: Abnormal Expression of miR-21 and miR-95 in cancer stem-like cells is associated with radioresistance of lung cancer. Cancer Invest 33(5): 165-171, 2015.

16 Sullivan JP, Spinola M, Dodge M, Raso MG, Behrens C, Gao B, Schuster K, Shao C, Larsen JE, Sullivan LA, Honorio S, Xie Y,
Scaglioni PP, DiMaio JM, Gazdar AF, Shay JW, Wistuba II and Minna JD: Aldehyde dehydrogenase activity selects for lung adenocarcinoma stem cells dependent on notch signaling. Cancer Res 70(23): 9937-9948, 2010.

17 Gao F, Zhou B, Xu JC, Gao X, Li SX, Zhu GC, Zhang XG and Yang C: The role of LGR5 and ALDH1A1 in non-small cell lung cancer: Cancer progression and prognosis. Biochem Biophys Res Commun 462(2): 91-98, 2015.

18 Alamgeer M, Ganju V, Szczepny A, Russell PA, Prodanovic Z, Kumar B, Wainer Z, Brown T, Schneider-Kolsky M, Conron M, Wright $G$ and Watkins DN: The prognostic significance of aldehyde dehydrogenase 1A1 (ALDH1A1) and CD133 expression in early stage non-small cell lung cancer. Thorax 68(12): 1095-1104, 2013.

19 Wei D, Peng JJ, Gao H, Zhang T, Tan Y and Hu YH: ALDH1 Expression and the prognosis of lung cancer: a systematic review and meta-analysis. Heart Lung Circ 24(8): 780-788, 2015.

20 Mizugaki H, Sakakibara-Konishi J, Kikuchi J, Moriya J, Hatanaka KC, Kikuchi E, Kinoshita I, Oizumi S, Dosaka-Akita H, Matsuno Y and Nishimura M: CD133 expression: a potential prognostic marker for non-small cell lung cancers. Int J Clin Oncol 19(2): 254-259, 2014.

$21 \mathrm{Li}$ Z: CD133: A stem cell biomarker and beyond. Exp Hematol Oncol 2(1): 17, 2013.

22 Bertolini G, D’Amico L, Moro M, Landoni E, Perego P, Miceli R, Gatti L, Andriani F, Wong D, Caserini R, Tortoreto M, Milione M, Ferracini R, Mariani L, Pastorino U, Roato I, Sozzi $\mathrm{G}$ and Roz L: Microenvironment-modulated metastatic CD133+/CXCR4+/EpCAM- lung cancer-initiating cells sustain tumor dissemination and correlate with poor prognosis. Cancer Res 75(17): 3636-3649, 2015.

23 Park E, Park SY, Sun PL, Jin Y, Kim JE, Jheon S, Kim K, Lee CT, Kim H and Chung JH: Prognostic significance of stem cellrelated marker expression and its correlation with histologic subtypes in lung adenocarcinoma. Oncotarget 7(27): 4250242512, 2016.

$24 \mathrm{Wu}$ H, Qi XW, Yan GN, Zhang QB, Xu C and Bian XW: Is CD133 expression a prognostic biomarker of non-small-cell lung cancer? A systematic review and meta-analysis. PLoS One 9: e100168, 2014

25 Woo T, Okudela K, Mitsui H, Yazawa T, Ogawa N, Tajiri M, Yamamoto T, Rino Y, Kitamura $\mathrm{H}$ and Masuda M: Prognostic value of CD133 expression in stage I lung adenocarcinomas. Int J Clin Exp Pathol 4(1): 32-42, 2010.

26 Okudela K, Woo T, Mitsui H, Tajiri M, Masuda M and Ohashi $\mathrm{K}$ : Expression of the potential cancer stem cell markers, CD133, $\mathrm{CD} 44, \mathrm{ALDH} 1$, and $\beta$-catenin, in primary lung adenocarcinoma-their prognostic significance. Pathol Int 62(12): 792-801, 2012.

27 Roudi R, Korourian A, Shariftabrizi A and Madjd Z: Differential expression of cancer stem cell markers ALDH1 and CD133 in various lung cancer subtypes. Cancer Invest 33(7): 294-302, 2015.

Received February 16, 2017

Revised April 11, 2017

Accepted April 19, 2017 